\title{
Long-term overall survival of endometrial carcinoma patients treated with high dose-rate brachytherapy with Norman-Simon catheters, vaginal brachytherapy and external beam radiotherapy
}

\author{
Jeno Palvolgyi* and Kofi Agyemang-Prempeh \\ Department of Oncoradiology, Petz County Teaching Hospital, Gyor, Zrinyi, Hungary
}

\begin{abstract}
Purpose and aims: To evaluate the 10-year overall survival rate of 128 patients with endometrial carcinoma grade 1-3, treated between 2000 and 2005 with our version of Heyman packing (HP), vaginal HDR brachytherapy (VBT) and external beam pelvic radiotherapy (EBRT).

Patients and methods: 23 inoperable patients (Group A.) received definitive irradiation with 3 to 7 fractions with HP and 13 of them EBRT. 57 patients, grade 1-2, (Group B) received two preoperative fractions performed with HP followed by surgery and two fractions VBRT. 48 patients, grade 2, with deep infiltrations (Group C) received additional EBRT.
\end{abstract}

Results: The 5-year overall survival rate (OS) was for Group A, B and C: 43.4\%, 87.7\% and 75\%, respectively, while the 10-year OS was for Group B and C: 78.9\% and $68.7 \%$.

Conclusion: Long-time OS data of endometrial cc. patients treated with Ir-192 HDR and modified Heyman packing method indicates the efficiency of our insertion technique and treatment planning.

\section{Introduction}

The classic protocol in treatment of endometrial carcinoma is to administer preoperative irradiation of the uterus followed by surgery. Except the medically inoperable patients, the recently accepted protocol is surgery with staging to determine the need for additional adjuvant therapy. The preoperative and definitive BT treatments are usualy performed with standard rigid applicators such as with intrauterine tandem [1], Y-shaped applicators [2,3] and with the modified Heyman packing method $[4,5]$.

At our institution, patients with adenocarcinoma of the endometrium, not suitable for surgery were treated between 2000 and 2005 with 2-7 fractions of our version of Heyman packing. Patients eligible for surgery received two fractions preoperative brachytherapy with HP method, followed by surgery and two fractions postoperative vaginal brachytherapy. Depending on the histology found, for the high-intermediate risk patients also external beam pelvic irradiation was administered.

\section{Marterials and methods}

\section{Our version of Heyman packing method}

The modified Heyman packing method, introduced by Herbolsheimer, usually is performed with 6-18 pieces of NormanSimon catheters, the heads of the catheters are uniformly distributed in the uterine cavity [4,5]. At our institution, endometrial insertions with smaller amount of Norman- Simon catheters (Nucletron, an Elekta company, Elekta AB, Stockholm, Sweden) are performed [6].
Depending on the dimension of the uterus, the number of catheters is between 4 or 7. Into the medium size uterus, five pieces of catheters with $6 \mathrm{~mm}$ diameter head are inserted usually. Another significant factor is the heads of each Norman-Simon catheters are positioned to the fundus uteri as illustrated in Figure 1. The aim of this catheter arrangement is to deliver proper dose to the most propable $(80 \%)$ location of endometrial cancer. The geometrical arrangement of the actual insertion often approximates the ideal one, but because of differences in location of catheters, individual treatment plan for each fraction is generated.

\section{Catheter insertion, imaging and treatment planning}

The patient lies on the dedicated treatment table (Air Liquide, France) in the lithotomy position. Intravenous systemic sedation followed by local anaesthesia (lidocaine $2 \%$ ) is used. The cervix is dilated to $10-12 \mathrm{~mm}$ using Hegar dilator, and the catheters are implanted into the uterine cavity. Since the treatment planning, even with reduced number of catheters is a time-consuming process, therefore after

Correspondence to: Jeno Palvolgyi, Department of Oncoradiology, Petz County Teaching Hospital, Gyor, Zrinyi, Hungary, Tel: + 3696507 900; E-mail: palvolgyij@petz.gyor.hu

Key words: brachytherapy, endometrial carcinoma, modified Heyman packing, overall survival

Received: September 12, 2017; Accepted: September 25, 2017; Published: September 28, 2017 


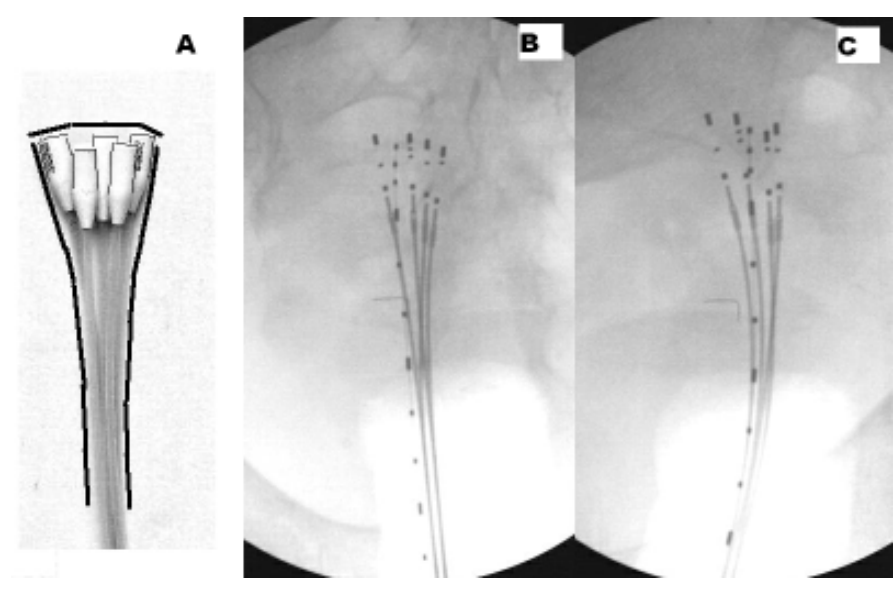

Figure 1. Typical catheter arrangement with our version of Heyman packing: five NormanSimon catheters were inserted into the uterus, all catheters' heads to the fundus (A), pair of posteri-anterior $(\mathrm{B})$ and posterior oblique $(\mathrm{C})$ reconstruction images.

finishing the catheter insertion, the patient is laid onto supine position and remains in the same position during the imaging, treatment planning and the irradiation. The biplane digital reconstruction images are obtained with a C-arm X-ray fluoroscopy unit (Mobilgil AR7, Giraldoni, Italy) located in the brachytherapy room.

The endometrial cancer patients are often obese, and the small power of the X-ray unit was not sufficient to obtain lateral images, therefore, instead of usual anteri-posterior (AP) and lateral image pair, we applied variable angle reconstruction method with digital images obtained by the $\mathrm{C}$-arm X-ray unit in BT treatment planning [7]. We obtain pairs of posteri-anterior (PA) and posterior-oblique (PO) images in the range between 135 and 150 degrees. In the PO images, the shield of the pelvic bones is lower compared to the lateral one and the visibility of the radio-opaque markers inserted into the catheters is significantly better. Typical pair of reconstruction images is illustrated in Figure 1B and $\mathrm{C}$. The reconstruction images are annotated with the imaging setup parameters and with the patient demographic data and with date and time stamp are converted to Dicom format. They are stored to pictorial archive and sent to the PLATO brachytherapy planning system (BPS), v13.7 (Nucletron) for applicator reconstruction. After digitizing the shape of the Norman-Simon catheters, in 3D view of reconstructed catheters, the laterally outermost left and right catheters are selected. Dose points at a distance of the uterine wall thickness are generated relative to the selected catheters. The dimensions of the uterus, including the wall thickness were obtained from MR or CT scans or from US performed prior to the first BT treatment. The wall thickness of the medium size uterus was typically $2 \mathrm{~cm}$. To fit the dose distributions to the dose points, the dwell time settings are optimized with the method 'on dose points and geometry'. Following the optimization, the dwell time settings are manually adjusted to improve the dose coverage at the uterine corners and fundus. The typical dose distribution is illustrated in Figure 2. The prescribed dose is $7 \mathrm{~Gy} / \mathrm{Fr}$ administered with the microSelectron V2 HDR after-loader (Nucletron).

\section{Vaginal brachytherapy}

Postoperative vaginal brachytherapy (VBT) treatments were performed with the single channel vaginal applicator (Nucletron) of 2 to $3.5 \mathrm{~cm}$ diameter. The prescribed dose was normalized and optimized for dose points in a distance of $0.5 \mathrm{~cm}$ apart from the applicator surface.

\section{External beam radiotherapy}

External beam radiotherapy (EBRT) to the pelvis were performed with AP and PA opposed fields with the Cobalt unit (T780C,
Theratronix, Canada) or with $18 \mathrm{MV}$ X-ray (Primus, Siemens, Germany). The common field borders were at the L4/5 inter-veterbral space superiorly, at the inferior border of the obturator foramen inferiorly, and $1.5-2.0 \mathrm{~cm}$ lateral to the inner bony margins of the true pelvis. Individual shields were inserted at the field corners. A total of $30-50$ Gy is delivered with 1.8 to 2 fractions 5 days a week. A midline block of $2 \mathrm{~cm}$ width is inserted to the treatment field after delivering $30 \mathrm{~Gy}$.

\section{Data collection for calculation of the overall survival time}

128 patients with endometrial carcinoma, grade 1-3 treated between 2000 and 2005 were considered, divided into three groups listed below. The HP treatment dates, the number of inserted NormanSimon catheters were obtained from the BT pictorial archive, while the description of the surgery, staging and histology founds were collected from the medical records. The survival status on 5th February 2015 or the exit date was provided by the Central Bureau of Electronic Public Services (Budapest, Hungary). The number of survival months was computed with the difference between the exit date and the first HP treatment date.

Group A: 23 patients were not eligible for hysterectomy because of aging (mean age: 74 years, range 60 to 80 ) and other chronic illnesses, received definitive radiotherapy. The $\mathrm{BT}$ treatments were performed with 5 to 7 fractions, one fraction/week schedule majority with HP. For 3 patients, 3 of 5 fractions were administered with the Y-shaped applicator (Nucletron) because of technical reason or uterine corner involvement. One patient was treated in 3 of 5 fractions with FletcherSuit applicator (Nucletron), because of cervical invasion. For 13 patients external beam radiotherapy for the pelvis was administered, among 7 of 13 received total dose of $44 \mathrm{~Gy}$ to $50 \mathrm{~Gy}$ with $1.8 \mathrm{~Gy} / \mathrm{Fr}$ while the others received $40 \mathrm{~Gy}$ with $2 \mathrm{~Gy} / \mathrm{Fr}$.

Group B.: 57 low risk endometrial cancer patients (mean age: 61 years, range 40 to 87 ) with less than $5 \mathrm{~mm}$ infiltration, grade $1-2$, received preoperative HP with 2 fractions. The dose for the outer uterine contour was $7 \mathrm{~Gy}$, one Fr/week schedule. Total hysterectomy and bilateral salpingo-oophrectomy (TAH-BSO) with or without pelvic lymphadenectomy was performed. Postoperative VBT treatments were administered with two or three fractions, $5.5 \mathrm{~Gy} / \mathrm{Fr}$ or $5 \mathrm{~Gy} / \mathrm{Fr}$ with one Fr/week schedule.

Group C: 48 intermediate risk endometrial cancer patients (mean age: 63 range 40 to 79), the majority grade 2 and with residual tumour

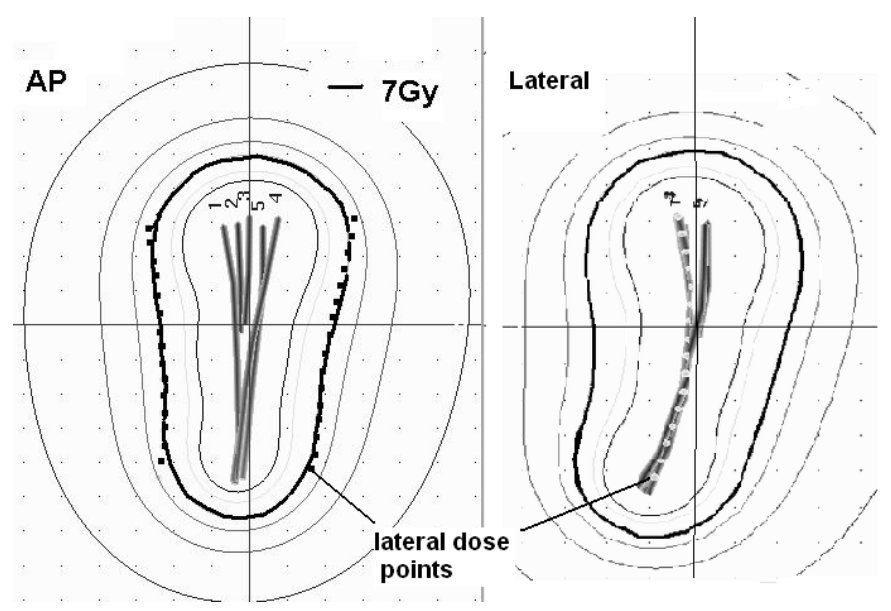

Figure 2. Anteri-posterior and lateral views of typical dose distribution optimized for dose points at a lateral distance of the uterine wall thickness from two selected catheters (\#1 and \#4). 
Palvolgyi J (2017) Long-term overall survival of endometrial carcinoma patients treated with high dose-rate brachytherapy with Norman-Simon catheters, vaginal brachytherapy and external beam radiotherapy

or deep infiltration were treated the same way as Group B. completed with pelvic EBRT. The majority of the patients received total dose of $40 \mathrm{~Gy}$ with $2 \mathrm{~Gy} / \mathrm{Fr}$, while for 8 patients, total dose of $50 \mathrm{~Gy}$ with $1.8 \mathrm{~Gy} /$ Fr was administered.

\section{Results}

The overall survival (OS) curves for Group A. B. and C. are illustrated in Figure 3. The 5-year OS was $43.4 \%$ (A), $87.7 \%$ (B) and $75 \%(C)$, while the 10 -year OS for Group B and C was $78.9 \%$ and $68.7 \%$ respectively as summarized in Table 1 .

\section{Discussion}

Trials in treatment of the endometrial carcinoma with brachytherapy were performed mainly with tandem [1] or Rotte applicator [2]. The earlier published survival data summarised by Weitmann, et al. [5] are pertained to LDR brachytherapy mainly. The 5 -year OS varied between $40 \%$ and $61 \%$ while the disease-free survival (DSS) was between $76 \%$ and $87 \%$. Only single study was published with Ir-192 HDR and modified Heyman packing method in definitive treatment [5]. The 4-year overall survival for a small number of patients (16) was $39 \%$, while the DSS was $100 \%$.

The significant factors of our insertion technique and treatment planning method are indicated in paragraph Materials and methods. The majority of HP insertions approximated the ideal catheter arrangement which is illustrated in Figure 1. In time of the treatments of our study, the sectional image guided brachytherapy was not available at our institution. We investigated the dose distribution of our HP insertions with 2D dosimetry. In our previous study, we compared the size of the small, medium and the large uterus prior and after the insertion of 4, 5 and 6 Norman-Simon catheters with $6 \mathrm{~mm}$ heads. No significant change in uterine size was found [8]. The dose distributions of ideal arrangements with 5 and 6 catheters showed significantly higher dose at the fundus uteri compared to the Y-shaped applicator's dose field. However, the dose plans were prepared with $2 \mathrm{D}$ dosimetry, the uterus dimension and the wall thickness obtained from diagnostic CT, MR scan or US found was incorporated into the treatment planning and individual plans were generated for each treatment fraction. Since the flexible catheters

Table 1. Summary of treatment details and 5 years and 10 years overall survival rates for groups A, B and C.

\begin{tabular}{|c|c|c|c|c|c|c|c|c|c|c|c|}
\hline $\begin{array}{c}\text { Age at } \\
\text { treatment }\end{array}$ & $\begin{array}{c}\text { Number of } \\
\text { patients }\end{array}$ & Grade & Treatment & Dose prescription & Applicator & VBT & \multicolumn{3}{|c|}{ EBRT } & 5 years OS & 10 Years OS \\
\hline $70[60-80]$ & $\begin{array}{l}19 \text { of } 23 \\
3 \text { of } 23 \\
1 \text { of } 23\end{array}$ & $1 \div 3$ & Definitive BT & $\begin{array}{c}5 \div 7 \mathrm{Fr} 7 \mathrm{~Gy} / \mathrm{Fr} \\
3 \text { of } 5 \mathrm{FR} \\
3 \text { of } 5 \mathrm{FR}\end{array}$ & $\begin{array}{c}\text { HP } \\
\text { Rotte } \\
\text { Fletcher }\end{array}$ & 03 of 23 & $\begin{array}{l}7 \text { of } 13 \\
6 \text { of } 13\end{array}$ & $\left|\begin{array}{c}44 \div 50 \\
40 \mathrm{GY}\end{array}\right|$ & $\begin{array}{l}1.8 \mathrm{~Gy} / \mathrm{Fr} \\
2 \mathrm{~Gy} / \mathrm{Fr}\end{array}$ & $43 \%$ & \\
\hline $60[40-87]$ & 57 & $1 \div 2$ & $\begin{array}{c}\text { Preoperative BT } \\
\text { Postoperative BT }\end{array}$ & $2 \mathrm{Fr} 7 \mathrm{~Gy} / \mathrm{Fr}$ & HP & $\begin{array}{l}2 \mathrm{Fr} 5.5 \mathrm{~Gy} / \mathrm{Fr} \\
\text { Or } 3 \mathrm{Fr} 5 \mathrm{~Gy} / \mathrm{Fr}\end{array}$ & & & & $87.7 \%$ & $75.0 \%$ \\
\hline 63 [40-69] & 48 & 2 & $\begin{array}{c}\text { Preoperative BT } \\
\text { Postoperative BT }\end{array}$ & $2 \mathrm{Fr} 7 \mathrm{~Gy} / \mathrm{Fr}$ & HP & $\begin{array}{l}2 \mathrm{Fr} 5.5 \mathrm{~Gy} / \mathrm{Fr} \\
\text { Or } 3 \mathrm{Fr} 5 \mathrm{~Gy} / \mathrm{Fr}\end{array}$ & $\begin{array}{l}42 \text { of } 50 \\
8 \text { of } 48\end{array}$ & $\begin{array}{l}40 \mathrm{GY} \\
50 \mathrm{GY}\end{array}$ & $\begin{array}{l}2 \mathrm{~Gy} / \mathrm{Fr} \\
1.8 \mathrm{~Gy} / \mathrm{Fr}\end{array}$ & $78.9 \%$ & $68.7 \%$ \\
\hline
\end{tabular}

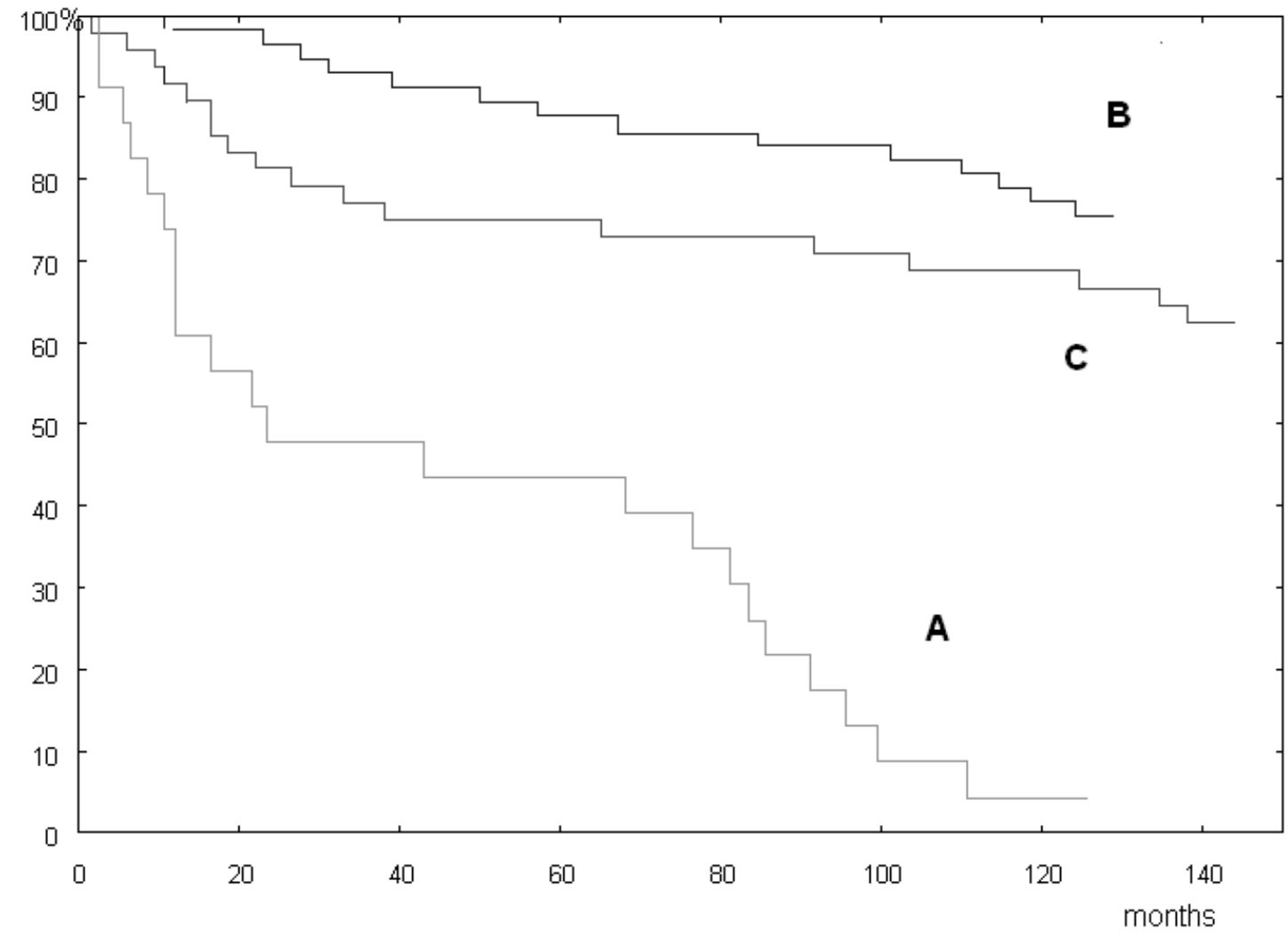

Figure 3. Overall survival rate of patients with definitive treatment (A) and OS of low and intermediate risk patients (B) and (C). 
Palvolgyi J (2017) Long-term overall survival of endometrial carcinoma patients treated with high dose-rate brachytherapy with Norman-Simon catheters, vaginal brachytherapy and external beam radiotherapy

followed the shape of the uterus, the dose points also followed the uterine contour and resulted in a proper dose coverage for the small and medium size uterus [6].

\section{Conclusion}

The 5-year OS values of present study belongs to the range of highest ones reported in literature indicate the efficiency of the applied insertion technique and dosimetry.

\section{Conflict of interest}

There is no conflict of interest in this paper.

\section{References}

1. Knocke TH, Kucera H, Weidinger B, Höller W, Pötter R (1997) Primary treatment of endometrial carcinoma with high-dose-rate brachytherapy: results of 12 years of experience with 280 patients. Int J Radiat Oncol Biol Phys 37: 359-365.
2. Coon D, Beriwal S, Heron DE, Kelley JL, Edwards RP, et al. (2008) High-dose-rate Rotte "Y" applicator brachytherapy for definitive treatment of medically inoperable endometrial cancer: 10-year. Int J Radiat Oncol Biol Phys 71: 779-783.

3. van der Steen-Banasik E (2014) Primary brachytherapy as a radical treatment for endometrial carcinoma. J Contemp Brachytherapy 6: 106-112.

4. Herbolsheiner M, Sauer O (1996) HDR Brachytherapy for Endometrial Cancer International Nucletron Radiotherapy Journal Special Report No. 8: 34-45.

5. Weitmann HD, Pötter R, Waldhaus C (2005) Treatment of endometrial carcinoma with 3D image-based high dose rate brachytherapy using modified Heyman packing: clinical experience and dose volume histogram analysis. Int J Radiat Biol Phys 62: 468-478.

6. Pálvölgyi J, Agyemang-Prempeh K (2010) Modified Heyman packing performed with small number of Simon-Norman catheters. J Contemp Brachytherapy 2: 33-36.

7. Pálvölgyi J (2006) To what extent can digital images obtained with a non-isocentric $\mathrm{C}$-arm be used for brachytherapy treatment planning in gynaecology. Radiother Oncol 67: 107-112.

8. Palvolgyi J (2006) Dose distribution of modified heyman packing. Phys Med 22: 127130. [Crossref]

Copyright: (C2017 Palvolgyi J. This is an open-access article distributed under the terms of the Creative Commons Attribution License, which permits unrestricted use, distribution, and reproduction in any medium, provided the original author and source are credited. 